\title{
Anomalies, effective action and Hawking temperatures of a Schwarzschild black hole in the isotropic coordinates
}

\author{
Shuang-Qing $\mathrm{Wu}^{1,2)}$, Jun-Jin Peng ${ }^{1)}$ and Zhan-Yue Zhao \\ 1) College of Physical Science and Technology, Central China Normal University, Wuhan, \\ Hubei 430079, People's Republic of China \\ 2) Institute of Particle Physics, Hua-Zhong Normal University, Wuhan, Hubei 430079, \\ People's Republic of China \\ E-mail: sqwu@phy.ccnu.edu.cn
}

\begin{abstract}
Motivated by the universality of Hawking radiation and that of the anomaly cancellation technique as well as that of the effective action method, we investigate the Hawking radiation of a Schwarzschild black hole in the isotropic coordinates via the cancellation of gravitational anomaly. After performing a dimensional reduction from the four-dimensional isotropic Schwarzschild metric, we show that this reduction procedure will, in general, result in two classes of two-dimensional effective metrics: the conformal equivalent and the inequivalent ones. For the physically equivalent class, the two-dimensional effective metric displays such a distinct feature that the determinant is not equal to the unity $(\sqrt{-g} \neq 1)$, but also vanishes at the horizon, the latter of which possibly invalidates the anomaly analysis there. Nevertheless, in this paper we adopt the effective action method to prove that the consistent energy-momentum tensor $T_{t}^{r}$ is divergent on the horizon but $\sqrt{-g} T_{t}^{r}$ remains finite there. Meanwhile, through an explicit calculation we show that the covariant energymomentum tensor $\widetilde{T}_{t}^{r}$ equals to zero at the horizon. Therefore the validity of the covariant regularity condition that demands that $\widetilde{T}_{t}^{r}=0$ at the horizon has been justified, indicating that the gravitational anomaly analysis can be safely extrapolated to the case where the metric determinant vanishes at the horizon. It is then demonstrated that for the physically equivalent reduced metric, both methods can give the correct Hawking temperature of the isotropic Schwarzschild black hole, while for the inequivalent one with the determinant $\sqrt{-g}=1$ it can only give half of the correct temperature. We further exclude the latter undesired result by taking into account the general covariance of the energy-momentum tensor under the isotropic coordinate transformation.
\end{abstract}

Keywords: Anomaly, Effective action, Hawking radiation, Black hole, Isotropic coordinate

Submitted to: Class. Quantum Grav.

PACS numbers: 04.62.+v, 04.70.Dy, 11.30.-j 


\section{Introduction}

Hawking radiation [1] is one of the most important theoretical discoveries in black hole physics, named after the surname of famous physicist Steven Hawking. The Hawking effect shows that a black hole is not really black, but can radiate thermally like a black body. Precisely speaking, Hawking radiation is the quantum effect of field in a classically curved background space-time with a future event horizon. It has a feature that the thermal radiation is determined universally by the properties of the horizon. There are several derivations of Hawking radiation. The original one presented by Hawking [1] when he quantized the scalar field theory in a static Schwarzschild space-time is undoubtedly the most direct method through which Hawking straightforwardly calculated the Bogoliubov coefficients between inand out-states of fields in a black hole background. This derivation, however, is very intricate and difficult to be generalized to more general cases. Later on, other attempts were made now and then.

Recently, there are two popular and relatively simple methods owing to Wilczek [2, 3, 4], attracting a lot of attention [5, 6, 7, 8, 9, 10, 11, 12, 13, 14, 15, 16]. One is the semiclassical tunnelling picture proposed by Parikh and Wilczek [2], and the other is the anomaly cancellation method advocated by Robinson and Wilczek (RW) [3, 4]. In the former method, Hawking radiation is visualized as a tunnelling process from the horizon and can be derived by calculating the semi-classical WKB amplitudes for classically forbidden paths. In the latter one, Hawking radiation can be understood as a compensating energy-momentum flux required to cancel the consistent gravitational anomaly at the horizon in order to preserve general covariance at the quantum level. Obviously, the RW's method is very universal since this kind of derivation of Hawking radiation via the viewpoint of the anomaly cancellation is only dependent on the anomaly taking place at the horizon.

In the further development, Iso et al. [4] extended the RW's method to investigate Hawking radiation in the case of a charged black hole, by considering gauge anomaly in addition to gravitational anomaly. In their work, the condition for consistent gauge and gravitational anomaly cancellation and regularity requirement of covariant anomalies at the horizon, together with the energy-momentum conservation law, determines Hawking fluxes of the charge and energy-momentum tensor. Subsequently, the treatment was further generalized [5] to the cases of rotating and charged black holes, where the rotation essentially plays the role of a $S O(2) \simeq U(1)$ gauge field. Since then, a lot of application [6, 7, 8, 9, 10, 11] of this method appeared and were devoted to investigating Hawking radiation of other different black objects in various dimensions, and all the obtained results demonstrated that the gauge current and energy-momentum tensor flux, required to cancel the consistent gauge and gravitational anomalies at the horizon, are exactly equal to that of Hawking radiation. These results once again show that Hawking radiation is universal and only depends on the property of the horizon. It appears that these studies successfully support that the RW's prescription is very universal. However, it should be pointed out that almost all of these efforts were limited to the case of the determinant $\sqrt{-g}=1$ of the effective diagonal metric in two dimensions. Based on the original work of [3, 4], the anomaly cancellation method was extended [8] to 
the most general case of two-dimensional non-extremal black hole metrics where $\sqrt{-g} \neq 1$. Nevertheless, the anomaly analysis presented in [8] had overlooked a peculiar case of which the determinant $\sqrt{-g}$ vanishes at the horizon.

It should be emphasized that the original anomaly cancellation method proposed in Refs. [3, 4] encompasses not only the consistent anomaly but also the covariant one. Quite recently, Banerjee and Kulkarni [12] suggested that this model can be further simplified by using only the covariant gauge and gravitational anomalies to derive Hawking radiation from charged black holes. Their simplification makes the anomaly analysis more economical and conceptually cleaner. An extension of their work to the case where the determinant $\sqrt{-g} \neq 1$ was done in [13]. Based upon these developments, some direct applications soon appeared in [14, 15].

Very recently, Banerjee and Kulkarni [16] advocated to use the covariant boundary condition and the covariant energy-momentum tensor to directly calculate the Hawking fluxes from charged black holes via the effective action method. The results obtained by them are in accordance with those obtained by the RW's method. They also established the connection of this approach with the calculations based on the Unruh vacuum. However, the equivalence of the effective action method and the covariant anomaly method needs to be further clarified in the very general case where $\sqrt{-g} \neq 1$.

Note however, in fact, that there is a subtlety in the anomaly analysis, namely, the validity of the RW's method implicitly assumes the regular behaviors of the dilaton field and the metric determinant at the horizon. In other words, the metric determinant should not vanish or diverge on the horizon. This issue had been neglected in the previous researches since almost all of them only deal with the trivial case where $\sqrt{-g}=1$. As will be demonstrated in this paper, the above assumption is apparently violated in the case for a Schwarzschild black hole in the isotropic coordinate system. Therefore it is necessary to investigate extensively the most general case where $\sqrt{-g} \neq 1$, in particular, the special case when $\sqrt{-g}$ vanishes at the horizon. The Schwarzschild black hole in the isotropic coordinates just provides such an example that its metric determinant vanishes at the horizon.

In this paper, we attempt to apply the gravitational anomaly method and the effective action method to study Hawking radiation of the Schwarzschild black hole in isotropic coordinate system. Although the same question had been previously studied [3, 16] in the standard Schwarzschild coordinates, the isotropic coordinates display such a distinct feature that the metric determinant of the reduced two-dimensional effective and physically equivalent metric is not equal to the unity $(\sqrt{-g} \neq 1)$, but also vanishes at the horizon, the latter of which possibly invalidates the anomaly analysis there when one applies it to investigate the Hawking radiation in such a coordinate system. The interest to the isotropic coordinates is that they play an important role in finding a large classes of supersymmetric black hole solutions in string theory. For example, the famous five-dimensional BMPV black hole solution [17] can be expressed in terms of isotropic coordinates. Our motivation for this investigation is also inspired from the fact that Hawking radiation is a universal quantum phenomenon and should not depend on the concrete choice of different coordinates, but it really relies on the property of the horizon. Correspondingly, the Hawking temperature derived in different coordinates 
should be given by the same value, and not be related with a concrete coordinate system. Our aim is intended to verify that the RW's method and the effective action method are unrelated with a different choice of coordinates.

After performing a dimensional reduction procedure from 4 to 2 dimensions, we show that the dimensional reduction technique will, in general, result in two classes of twodimensional effective metrics: the equivalent and the inequivalent ones. We define the physically equivalent reduced metrics as those that neither change the rank of the zeros of the components $g_{t t}$ and $g^{r r}$ of the original four-dimensional metric, nor flip their signs. Otherwise we shall call them the in-equivalent classes. For the physically equivalent class, the effective two-dimensional metric displays such a distinct feature that the metric determinant is not equal to the unity $(\sqrt{-g} \neq 1)$, but also vanishes at the horizon, the latter of which possibly gives rise to a doubt about the reliability of a direct application of the anomaly analysis there.

In order to prove that the anomaly analysis can be safely extrapolated to the situation when the metric determinant vanishes at the horizon, we derive the consistent energymomentum tensor given by the effective action [5, 16, 18, 19] in the Unruh vacuum state. We find that the energy-momentum tensor $T_{t}^{r}$ is finite at spatial infinity but diverges on the horizon, therefore $\sqrt{-g} T_{t}^{r}$ rather than $T_{t}^{r}$ itself is regular on the horizon because $\sqrt{-g}$ vanishes there. We verify the regularity condition via explicitly calculating the covariant energy-momentum tensor and showing that both $\widetilde{T}_{t}^{r}$ and $\sqrt{-g} \widetilde{T}_{t}^{r}$ vanish at the horizon. This means that the validity of the anomaly analysis at the horizon can be guaranteed and the RW's method can be safely extrapolated to the case when $\sqrt{-g}$ vanishes at the horizon. We further demonstrate that for the physically equivalent reduced metric, both the effective action method and the RW's method can derive the correct Hawking temperature of the Schwarzschild black hole in the isotropic coordinates, which is exactly in accordance with Hawking's original result; while for the inequivalent one with $\sqrt{-g}=1$ it can only give a temperature with its value just being one-half of the correct one originally derived by Hawking. The latter result is undesired and can be further excluded by taking into account the covariance of the energymomentum tensor under the isotropic coordinate transformation.

Our paper is outlined as follows. In section 2, we derive the line element of a Schwarzschild black hole in the isotropic coordinates from the standard coordinates by means of the isotropic coordinate transformation and calculate its surface gravity via the standard formulae. We compare the difference between these two coordinate systems in detail. In section 3, we present the dimensional reduction of the massless scalar field theory in the isotropic Schwarzschild space-time and demonstrate that this reduction procedure will, in general, result in two classes of two-dimensional effective metrics: the equivalent and the inequivalent ones. In section 4, we compute the consistent energy-momentum tensor given by the effective action [5, 16, 18, 19] in the Unruh vacuum state and show that $T_{t}^{r}$ diverges on the horizon, but $\sqrt{-g} T_{t}^{r}$ is finite there. Meanwhile, the covariant energy-momentum tensor is explicitly calculated and shown to be equal to zero at the horizon, verifying the validity of the covariant regularity condition $\widetilde{T}_{t}^{r}=0$ at the horizon. This fact confirms us that the validity of the gravitational anomaly analysis at the horizon is guaranteed and the RW's method can be safely extrapolated to the case where the metric determinant vanishes at the horizon. Section 
5 is devoted to deriving the Hawking temperature via the cancellation of the consistent or covariant anomaly at the horizon. It is shown that for the physically equivalent reduced metric, in which the resultant determinant vanishes at the horizon, both the effective action method and the anomaly cancellation method can give a temperature of the isotropic Schwarzschild black hole, which is just the correct one derived by Hawking in his original work; while for the inequivalent class, with which the determinant $\sqrt{-g}=1$ in the two-dimensional effective background field theory, both methods can only derive a temperature that is one-half of the original one given by Hawking. Finally we further exclude this incorrect result by taking into account the general covariance of the energy-momentum tensor. Our results show that the Hawking temperature have the same value in different coordinate systems. In addition, the equivalence between the effective action method and the RW's method has also been established. The last section 6 ends up with a summary of our main results and presents our conclusions.

\section{The Schwarzschild black hole: the isotropic coordinates versus the standard ones}

There are several different forms for the metric of a Schwarzschild black hole. Among them, the most popular one is expressed in the standard Schwarzschild coordinate system as follows

$$
d s^{2}=-\left(1-\frac{2 M}{\hat{r}}\right) d t^{2}+\frac{d \hat{r}^{2}}{1-2 M / \hat{r}}+\hat{r}^{2} d \Omega^{2},
$$

where $M$ is the Arnowitt-Deser-Misner (ADM) mass of the black hole, $d \Omega^{2}$ is the metric on the unit two-sphere. It is well known that an event horizon of the black hole is determined by $g^{\hat{r} \hat{r}}=0$ while a Killing horizon is given by $g_{t t}=0$. Obviously, the Schwarzschild black hole has an event horizon that coincides with the Killing horizon at $\hat{r}_{H}=2 M$ where the metric becomes singular.

For the question in which we interested here, we shall consider the Schwarzschild black hole in the isotropic coordinates. After performing a coordinate transformation

$$
\hat{r}=r(1+M / 2 r)^{2},
$$

the line element (1) then becomes

$$
d s^{2}=-\frac{(1-M / 2 r)^{2}}{(1+M / 2 r)^{2}} d t^{2}+\left(1+\frac{M}{2 r}\right)^{4}\left(d r^{2}+r^{2} d \Omega^{2}\right),
$$

for which the metric determinant is

$$
\sqrt{-g_{4}}=r^{2}\left(1-\frac{M}{2 r}\right)\left(1+\frac{M}{2 r}\right)^{5} \sin \theta .
$$

In this isotropic coordinate system, the black hole now has only a Killing horizon which is located at $r_{H}=M / 2$, corresponding to $\hat{r}_{H}=2 M$ in the standard coordinate system. We can see that the metric (3) is regular at $r_{H}=M / 2$. Now we want to calculate the temperature of the back hole in the isotropic coordinates. The surface gravity can be computed in the standard manner and is given by

$$
\kappa=-\left.\frac{1}{2} \sqrt{\frac{g^{r r}}{-g_{t t}}} \frac{\partial g_{t t}}{\partial r}\right|_{r=r_{H}}=\frac{1}{4 M},
$$


so the corresponding Hawking temperature is

$$
T=\frac{\kappa}{2 \pi}=\frac{1}{8 \pi M},
$$

which is exactly the same one as that calculated in the standard coordinate system. Therefore, one can draw a conclusion that Hawking temperature should be determined by the properties of the horizon but unrelated with the choice of different coordinates. It is anticipated that an analogous universality can apply to the effective action method and the gravitational anomaly method.

There are some common characters shared by the black hole space-time in both metric forms, which can be summarized as follows. Both line elements are asymptotically flat at spatial infinity; The parameter $M$ is the ADM mass in both metrics; and the surface gravity has the same expression $\kappa=1 /(4 M)$ in both coordinate systems.

However, there exist a lot of explicit differences between these two coordinates in differen aspects such as, the type of horizons, properties of the singularity and the metric determinant, the zeros and signs of the temporal and radial metric components, etc. In the standard coordinates, the black hole horizon $\hat{r}_{H}=2 M$ is both an event horizon and a Killing horizon; the determinant $\sqrt{-\hat{g}_{4}}=\hat{r}^{2} \sin \theta$ is regular everywhere; the zeros of the components $g_{t t}$ and $g^{\hat{r} \hat{r}}$ are the same, both metric components flip their signs at the horizon. By contrast, in the isotropic coordinates, the location $r_{H}=M / 2$ is only a Killing horizon; the determinant $\sqrt{-g_{4}}=\sqrt{-\hat{g}_{4}} \zeta(r)$ vanishes at the horizon; the zeros of the components $g_{t t}$ and $g^{r r}$ are different, and their signs do not change at $r_{H}=M / 2$. These differences can be attributed to the fact that the isotropic coordinate transformation (2) has changed the order of zeros of the metric components and contributed to the metric determinant a Jacobi factor

$$
\zeta(r) \equiv \frac{\partial \hat{r}}{\partial r}=1-\frac{M^{2}}{4 r^{2}} .
$$

Finally, the asymptotic behaviors of the tortoise coordinates defined in both systems are different. In the standard Schwarzschild coordinates, the tortoise coordinate is defined as

$$
\hat{r}_{*}=\int \frac{\hat{r} d \hat{r}}{\hat{r}-2 M} \simeq 2 M \ln (\hat{r}-2 M) \equiv \frac{1}{2 \kappa} \ln \left(\hat{r}-\hat{r}_{H}\right),
$$

while it is defined in the isotropic coordinates via

$$
r_{*}=\int(1+M / 2 r)^{3} \frac{r}{r-M / 2} d r \simeq 4 M \ln (r-M / 2) \equiv \frac{1}{2 \kappa} \ln \left(r-r_{H}\right)^{2} .
$$

Their difference can be seen more clearly via finding the inverse transformation of Eq. (2), namely

$$
2 r=\hat{r}-M+\sqrt{\hat{r}^{2}-2 M \hat{r}}
$$

from which we get

$$
\sqrt{\hat{r}^{2}-2 M \hat{r}}=r-M^{2} / 4 r, \quad \hat{r}-M=r+M^{2} / 4 r .
$$

The above coordinate transformation maps the exterior region $\hat{r} \geq 2 M$ to the domain $r \geq M / 2$. Near the horizon $\hat{r}_{H}=2 M$ ( or $r_{H}=M / 2$ ), the asymptotic behaviors of these two coordinates are related by

$$
(r-M / 2)^{2} \simeq M(\hat{r}-2 M) / 2 .
$$


In addition, it is necessary to emphasize that the rank of the singularity (namely, the order of zeros of the metric component $g_{t t}$ ) has been changed under the isotropic coordinate transformation. That is, a simple singular point in the standard Schwarzschild coordinate system now becomes a rank-two singular point in the isotropic coordinate system.

\section{Dimensional reduction: the equivalent versus the in-equivalent classes}

Since the anomaly cancellation method proposed in Ref. [3] involves a dimensional reduction of the space-time from higher dimensions to two dimensions, therefore it is crucial to obtaining a reduced two-dimensional effective metric that inherits most fundamental properties of the higher-dimensional one. In this section, we shall show that the dimensional reduction procedure will yield two classes of two-dimensional effective background metrics: the conformal equivalent and the inequivalent ones. We present our definition for the equivalent and the inequivalent metrics, and demonstrate that even for the physically equivalent classes, the reduced two-dimensional effective metrics are not uniquely determined in the process of dimensional reduction, since they can differ by a regular conformal factor.

Before adopting the RW's method and the effective action method to investigate Hawking radiation from the isotropic Schwarzschild black hole, we now perform a dimension reduction in the isotropic coordinate system. Considering a massless scalar field in the background metric (3), the action can be written as

$$
\begin{aligned}
S[\varphi]= & -\frac{1}{2} \int d^{4} x \sqrt{-g} g^{\mu \nu} \partial_{\mu} \varphi \partial_{\nu} \varphi=\frac{1}{2} \int d^{4} x \sqrt{-g} \varphi \square \varphi \\
= & \frac{1}{2} \int d t d r d \theta d \phi r^{2} \sin \theta \varphi\left\{-\frac{(1+M / 2 r)^{7}}{1-M / 2 r} \partial_{t}^{2}\right. \\
& \left.+\frac{1}{r^{2}} \partial_{r}\left[\left(r^{2}-\frac{M^{2}}{4}\right) \partial_{r}\right]+\frac{1}{r^{2}}\left(1-\frac{M^{2}}{4 r^{2}}\right) \Delta_{\Omega}\right\} \varphi \\
= & \frac{1}{2} \sum_{l m} \int d t d r \varphi_{l m}\left\{-\frac{r^{2}(1+M / 2 r)^{7}}{1-M / 2 r} \partial_{t}^{2}+\partial_{r}\left[\left(r^{2}-\frac{M^{2}}{4}\right) \partial_{r}\right]\right. \\
& \left.+l(l+1)\left(1-\frac{M^{2}}{4 r^{2}}\right)\right\} \varphi_{l m} .
\end{aligned}
$$

where $\Delta_{\Omega}$ is the angular Laplace operator on the unit two-sphere, with the eigenvalue $l(l+1)$. In the last line, we have performed a partial wave decomposition $\varphi=\sum_{l m} \varphi_{l m}(t, r) Y_{l m}(\theta, \phi)$ in terms of the spherical harmonic functions.

Keeping the dominant terms in the vicinity of the horizon $\left(r_{H}=M / 2\right)$, the action is then simplified as

$$
S[\varphi] \simeq \frac{1}{2} \sum_{l m} \int d t d r r^{2} \varphi_{l m}\left[-\frac{(1+M / 2 r)^{7}}{1-M / 2 r} \partial_{t}^{2}+\left(1-\frac{M^{2}}{4 r^{2}}\right) \partial_{r}^{2}\right] \varphi_{l m} .
$$

The above near-horizon limit suggests that the action can be effectively replaced by the following one

$$
S[\varphi] \equiv \frac{1}{2} \sum_{l m} \int d t d r \Psi \varphi_{l m}\left[-\frac{1}{\sqrt{f h}} \partial_{t}^{2}+\sqrt{f h} \partial_{r}^{2}\right] \varphi_{l m},
$$


in a two-dimensional space-time with the effective metric

$$
d s^{2}=-f(r) d t^{2}+h(r)^{-1} d r^{2} .
$$

This indicates that the scalar field theory in the vicinity of the event horizon of the original four-dimensional black hole space-time (3) can be effectively described by an infinite collection of massless scalar fields in the two-dimensional effective background metric (16).

Comparing Eqs. (14) with (15), we get

$$
\frac{\Psi}{\sqrt{f h}}=r^{2} \frac{(1+M / 2 r)^{7}}{1-M / 2 r}, \quad \Psi \sqrt{f h}=r^{2}-\frac{M^{2}}{4} .
$$

From these equations, we can solve the dilaton factor as $\Psi=r^{2}(1+M / 2 r)^{4}$, and obtain

$$
\sqrt{f h}=\frac{1-M / 2 r}{(1+M / 2 r)^{3}} .
$$

With the help of the metric determinant $\sqrt{-g}=\sqrt{f / h}=\xi(r), f(r)$ and $h(r)$ can be obtained as follows

$$
f=\frac{1-M / 2 r}{(1+M / 2 r)^{3}} \xi(r), \quad h=\frac{1-M / 2 r}{(1+M / 2 r)^{3} \xi(r)} .
$$

Thus the reduced two-dimensional effective metric can be written as a conformal form as

$$
d s^{2}=\xi(r)\left[-\frac{1-M / 2 r}{(1+M / 2 r)^{3}} d t^{2}+\frac{(1+M / 2 r)^{3}}{1-M / 2 r} d r^{2}\right],
$$

with $\sqrt{-g}=\xi(r)$ acting as a conformal factor.

At this stage, we have only set the dilaton field as a regular function and left the metric determinant to be determined. Obviously, this implies that the resultant two-dimensional effective metrics are not unique. Thus, the dimension reduction procedure leaves us a room to choose many different expressions for the conformal factors, which can lead to several different results.

It is now a position to determine the concrete expression for the metric determinant $\sqrt{-g}=\xi(r)$. Note that its general expression assumes a splitting form

$$
\sqrt{-g}=\xi(r)=(1-M / 2 r)^{n} \chi(r),
$$

where the power $n$ is an integer, and $\chi(r)$ is an arbitrary regular function satisfying

$$
\chi\left(r=r_{H}\right) \neq 0, \infty ; \quad \chi(r \rightarrow \infty)=1 .
$$

The asymptotic behavior of the determinant at the horizon is mainly controlled by the power $n$. The latter condition means that $\sqrt{-g}=1$ when $r \rightarrow \infty$. The reason for this assumption will be explained later.

Now we propose our definition for the equivalent and the inequivalent classes of the reduced two-dimensional effective metrics.

Proposition: The physically equivalent classes for the reduced two-dimensional effective metrics are those that neither change the zeros of the components $g_{t t}$ and $g^{r r}$ of the original space-time metric (3), nor flip their signs at the horizon. Otherwise they shall be called the 
in-equivalent classes $\$$ The equivalent effective metrics are also conformal equivalent, up to a regular conformal factor $\chi(r)$ that satisfies the conditions (22).

According to the above definition, we can classify the reduced two-dimensional metrics (20) into two types:

(I) Conformal equivalent metrics: To ensure the physical equivalence of the reduced two-dimensional metric with the original four-dimensional one, the zeros and signs of the $g_{t t}$ and $g^{r r}$ components of the metric (3) should not be changed in the process of dimensional reduction. To retain the zeros and signs of the components $g_{t t}$ and $g^{r r}$ of the original metric (3), one must take $n=1$ for these equivalent classes. Thus the line element is

$$
d s^{2}=\chi(r)\left[-\frac{(1-M / 2 r)^{2}}{(1+M / 2 r)^{3}} d t^{2}+(1+M / 2 r)^{3} d r^{2}\right]
$$

with the determinant $\sqrt{-g}=\chi(r)[1-M /(2 r)]$ in which the regular function $\chi(r)$ acts like a conformal factor.

At this moment, the metric (23) is still not completely determined since $\chi(r)$ can be an arbitrary regular function as long as it satisfies the conditions (22). As such, the resultant two-dimensional effective metrics for the equivalent classes are not unique in the process of dimensional reduction, but are conformal equivalent under a regular conformal transformation. In other words, they are merely determined, up to a regular conformal factor.

We now explain the reason why one should demand that $\sqrt{-g}=1$ when $r \rightarrow \infty$. This is because the surface gravity $\kappa$ and $\sqrt{-g} T_{t}^{r}$ are conformal invariant physical quantities in two dimensions, so one must demand $\chi(r \rightarrow \infty)=1$ to obtain consistent physical results of the Hawking fluxes under a regular conformal transformation. In addition, this condition is also compatible with the asymptotically flatness of the metric at spatial infinity.

There are a lot of regular functions for $\chi(r)$ that satisfy the conditions (22). One natural choice is $\chi(r)=1+M /(2 r)$ so that $\sqrt{-g}=\zeta(r)=1-M^{2} /\left(4 r^{2}\right)$, then the line element becomes

$$
d s^{2}=-\frac{(1-M / 2 r)^{2}}{(1+M / 2 r)^{2}} d t^{2}+\left(1+\frac{M}{2 r}\right)^{4} d r^{2}
$$

which is exactly the $(t-r)$-sector of the original line element (3).

Alternatively, one can, for example, choose $\chi(r)=[1+M /(2 r)]^{-3}$ so that the reduced metric is

$$
d s^{2}=-\frac{(1-M / 2 r)^{2}}{(1+M / 2 r)^{6}} d t^{2}+d r^{2} .
$$

This line element differs from (24) by a regular conformal factor $[1+M /(2 r)]^{4}$. So even for the physically equivalent classes, the two-dimensional effective metrics, which are reduced from the four-dimensional space-time, need not to be the $(t-r)$-sector of the latter. This is different from the observation [3], where the dimension reduction keeps the form of the $(t-r)$-sector unchanged.

$\$$ In the case of a rotating black hole, the same question should be addressed in the dragging coordinate system. See for example, the first reference in [14]. 
It should be noted that the above two metrics are conformal equivalent, both of them give the consistent results in the anomaly analysis. However in both cases, $\sqrt{-g}=0$ at $r_{H}=M / 2$, $\sqrt{-g}=1$ at spatial infinity, and $\sqrt{-g} \neq 1$ elsewhere. That the metric determinant vanishes at the horizon will give rise to a doubt about the reliability of the anomaly analysis there.

Most related to the RW's method is to compute the surface gravity of the resultant twodimensional effective metric so as to reproduce the Hawking temperature of the original fourdimensional black hole. For the equivalent classes of the reduced two-dimensional metrics, the above two line elements differ by a regular conformal factor. Their surface gravities can be computed by making use of the standard method and are found to be the same value as that presented in the four-dimensional case, so the Hawking temperature is

$$
T=\frac{\kappa}{2 \pi}=\left.\frac{1}{4 \pi} \sqrt{h / f} \partial_{r} f\right|_{r_{H}}=\frac{1}{8 \pi M},
$$

which is just the standard value originally calculated by Hawking.

As will be seen later, both the RW's method and the effective action method can also derive the same correct Hawking temperature for the conformal equivalent two-dimensional metrics. However, a question that arises in the anomaly analysis is whether it is still valid for the case when $\sqrt{-g}=0$ at $r_{H}=M / 2$. In the next two sections, we will pay our attention to show that the anomaly analysis can be safely extrapolated to the situation when the metric determinant vanishes at the horizon. A key observation is to show that $\sqrt{-g} T_{t}^{r}$ rather than $T_{t}^{r}$ itself is finite on the horizon and $\widetilde{T}_{t}^{r}=0$ at the horizon.

(II) In-equivalent metrics: The metrics for the in-equivalent classes are those all that obey $n \neq 1$. In this case, one can arbitrarily select the conformal factor as long as it does not take $n=1$. For example, one can simply choose $\sqrt{-g}=\xi(r)=1$ so that

$$
d s^{2}=-\frac{1-M / 2 r}{(1+M / 2 r)^{3}} d t^{2}+\frac{(1+M / 2 r)^{3}}{1-M / 2 r} d r^{2} .
$$

With this choice of the conformal factor, the metric determinant is well-behaved everywhere, in particular, it is regular on the horizon. This feature is especially suitable to do the analysis via the anomaly cancellation method.

On the other hand, one can also select the line element to be that of the optical space

$$
d s^{2}=-d t^{2}+\frac{(1+M / 2 r)^{6}}{(1-M / 2 r)^{2}} d r^{2},
$$

for which the determinant will diverge on the horizon. Besides, other choice of the conformal factor is also possible. But at this moment, we only restrict ourself to the two cases listed above.

The temperature for the metric (26) with $f=h=[1-M /(2 r)] /[1+M /(2 r)]^{3}$ is

$$
T=\frac{\kappa}{2 \pi}=\left.\frac{1}{4 \pi} \partial_{r} f\right|_{r_{H}}=\frac{1}{16 \pi M}
$$

which is one-half of the value of Hawking's original result. On the other hand, the temperature calculated for the optical space is null, a rather different result. The same undesired result can also derived via the anomaly cancellation technique and the effective action method. So it turns out that the in-equivalent classes for the reduced metrics are physical meaningless, since 
all of them can not correctly reproduce the standard value for the Hawking temperature of a Schwarzschild black hole. Therefore, these types of the reduced metrics are undesirable in our discussions made below.

Before ending up with this section, let us briefly summarize the main results obtained in this section. We have generally shown that the original four-dimensional space-time (3) can be effectively reduced to two typically different kinds of two-dimensional metrics: the equivalent and the in-equivalent ones. For the physically equivalent two-dimensional metrics, we propose that the process of dimension reduction should make no change in the rank of the singularity of the original space-time metric, otherwise the resultant metrics belong to the in-equivalent classes. Note that the differences between the two-dimensional line elements (24) and (26) resemble much like those in the four-dimensional case listed in the last section. It should be emphasized that even for the equivalent classes, the reduced two-dimensional effective metrics need not to be completely identical with, but conformal to the $(t-r)$-sector of the original four-dimensional isotropic line element, up to a regular conformal factor. Only for the conformal equivalent classes can one derive the same correct temperature as that given by Hawking.

The above issue have not been exploited in the previous literature. In fact, the same technical question also exists in the dimensional reduction of the four-dimensional Schwarzschild black hole in the standard coordinates. To see this point more clearly, let us consider the near-horizon limit of the action of a massless scalar field

$$
S[\varphi] \simeq \frac{1}{2} \sum_{l m} \int d t d \hat{r} \hat{r}^{2} \varphi_{l m}\left[\frac{-1}{1-2 M / \hat{r}} \partial_{t}^{2}+\left(1-\frac{2 M}{\hat{r}}\right) \partial_{\hat{r}}^{2}\right] \varphi_{l m},
$$

which suggests it can be replaced by an effective action in the two-dimensional reduced metric $d \hat{s}^{2}=\sqrt{-\hat{g}} d s^{2}$, where $\sqrt{-\hat{g}}$ acts as a conformal factor and the line element

$$
d s^{2}=-\left(1-\frac{2 M}{\hat{r}}\right) d t^{2}+\frac{d \hat{r}^{2}}{1-2 M / \hat{r}},
$$

is the $(t-r)$-sector of the four-dimensional Schwarzschild space-time in the standard coordinates [4].

For the physically equivalent classes, one must impose that $\sqrt{-\hat{g}} \neq 0$ at $\hat{r}_{H}=2 M$ and $\sqrt{-\hat{g}}=1$ when $\hat{r} \rightarrow \infty$. The reduced two-dimensional metrics are not unique but are conformal equivalent. They need not to be in exact agreement with the $(t-r)$-sector of the fourdimensional metric, but a simple choice for the conformal factor is $\sqrt{-\hat{g}}=1$, which can reach to this end [3]. In addition, it is easy to find that the two-dimensional effective metric (24) can be obtained from (29) via the isotropic coordinate transformation (2), while the metric (26) can not.

In the remaining sections, we will demonstrate that in the case of a physically equivalent metric, both the effective action method and the gravitational anomaly cancellation method can give a temperature, which is exactly the same as the Hawking's original one. But for the in-equivalent metrics, the RW' method can not derive the correct Hawking temperature. For instance, in the case of a two-dimensional metric (26) with $\sqrt{-g}=1$, both methods give a temperature which is only one-half of the Hawking's standard one. In the remainder of this 
paper, we shall only focus on the case of a physically equivalent metric for which we must deal with the situation when the metric determinant vanishes at the horizon.

\section{Effective action, Unruh vacuum and Hawking flux}

In the preceding section, we have obtained, by means of the dimensional reduction, the physically equivalent classes of the resultant two-dimensional metrics which are conformal, by a regular conformal factor, to the $(t-r)$-sector of the four-dimensional Schwarzschild black hole in the isotropic coordinates.

From now on, we shall base upon our discussions in terms of a two-dimensional effective metric (24) written in the general form (16) as

$$
d s^{2}=-f(r) d t^{2}+h(r)^{-1} d r^{2},
$$

where

$$
f=\frac{(1-M / 2 r)^{2}}{(1+M / 2 r)^{2}}, \quad h=\frac{1}{(1+M / 2 r)^{4}} .
$$

The meter determinant is given by

$$
\sqrt{-g}=\sqrt{f / h}=1-\frac{M^{2}}{4 r^{2}} .
$$

It is obvious that $\sqrt{-g}=0$ at $r=r_{H}=M / 2, \sqrt{-g}=1$ as $r \rightarrow \infty$, and $\sqrt{-g} \neq 1$ elsewhere.

For later convenience, we denote the surface gravity at any point $r$ as

$$
K=\frac{1}{2} \sqrt{h / f} f^{\prime}=\frac{M r^{2}}{(r+M / 2)^{4}},
$$

where a prime $\left(I \equiv \partial_{r}\right)$ denotes the derivative with respect to the radial coordinate, here and hereafter. We also introduce the antisymmetric tensors $\varepsilon^{\mu v}=\varepsilon^{\mu \nu} / \sqrt{-g}$ and $\varepsilon_{\mu v}=\sqrt{-g} \varepsilon_{\mu \nu}$ in terms of the antisymmetric tensor density $\varepsilon^{\mu \nu}$ with the convention $\varepsilon^{t r}=\varepsilon_{r t}=1$.

The scalar curvature in the background (16) reads

$$
R=-2 \sqrt{h / f} K^{\prime}=\frac{4 M r^{3}}{(r+M / 2)^{6}} .
$$

Note that both $K$ and $R$ vanish at spatial infinity, and remain finite at the horizon $\left(r_{H}=M / 2\right)$. The surface gravity at the horizon is given by $\kappa=\lim _{r \rightarrow r_{H}} K=1 /(4 M)$.

As mentioned before, the validity of the RW's method might not be guaranteed at the horizon as the metric determinant vanishes there. In order to justify the validity of the anomaly analysis at the horizon, we have to prove that $T_{t}^{r}$ diverges on the horizon but $\sqrt{-g} T_{t}^{r}$ remains finite there. Our strategy will be to calculate the anomaly induced stress tensor in the Unruh vacuum by using the trace anomaly in the context of a two-dimensional black hole with the effective metric (24). The main reason for this is because the boundary condition adopted in the gravitational anomaly method is consistent with the Unruh vacuum, and the anomaly cancellation method with this boundary condition gives the same result as the one derived using the trace anomaly method. What is more, we also explicitly compute the covariant 
energy-momentum tensor and show that it indeed vanishes at the horizon, thus verifying the validity of the regularity condition $\widetilde{T}_{t}^{r}=0$ at the horizon.

In the case of a Schwarzschild black hole, there are a lot of work [18, 19] to derive Hawking flux from effective actions [5, 16, 18, 19] in the two-dimensional effective background. Although the two-dimensional effective theory contains a dilaton field, the effect of dilaton does not change the property of $(r, t)$-component of the energy-momentum tensor and accordingly the Hawking flux is independent of the dilaton background. Once we impose the boundary condition, the value of the energy flux is determined only by the value of anomalies at the horizon. Therefore in the following, it will suffice for us to make use of the effective action to calculate the energy flux in the two-dimensional effective metric without dilaton backgrounds.

\subsection{Consistent energy-momentum tensor and Hawking flux}

It is known that the two-dimensional trace anomaly is

$$
T_{\mu}^{\mu}=-\frac{R}{24 \pi} \text {. }
$$

The quantum anomaly induced effective action [5, 19] can be obtained by functional integration of this trace anomaly (33). It is the well-known Polyakov-Liouville's non-local action [20]

$$
\Gamma[g]=-\frac{1}{96 \pi} \int d^{2} x \sqrt{-g} R \square^{-1} R,
$$

where $\square$ is the covariant Laplacian operator in two dimensions.

We recognize this effective action reflects the structure of the conformal anomaly of Eq. (33). This action is non-local, but it can be transformed to a local form by introducing an auxiliary field $\psi[5,19]$

$$
\Gamma[\psi, g]=-\frac{1}{96 \pi} \int d^{2} x \sqrt{-g}(-\psi \square \psi+2 \psi R),
$$

where the auxiliary field $\psi$ satisfies the equation $\square \psi=R$.

The quantum anomaly induced stress tensor is defined via the variation of this effective action with respect to $g^{\mu \nu}$ as follows

$$
T_{\mu \nu}=\frac{2}{\sqrt{-g}} \frac{\delta \Gamma[\psi, g]}{\delta g^{\mu \nu}}=-\frac{1}{48 \pi}\left[\nabla_{\mu} \psi \nabla_{\nu} \psi-2 \nabla_{\mu} \nabla_{\nu} \psi+g_{\mu \nu}\left(2 R-\frac{1}{2} \nabla^{\rho} \psi \nabla_{\rho} \psi\right)\right] .
$$

This stress tensor obeys the trace anomaly equation (33) and the conservation law

$$
T_{\mu}^{\mu}=-\frac{R}{24 \pi}, \quad \nabla_{\mu} T_{v}^{\mu}=0 .
$$

For the two-dimensional effective metric (16), the equation of motion for the auxiliary field $\psi$ becomes

$$
-\frac{1}{\sqrt{f h}} \partial_{t}^{2} \psi+\partial_{r}\left(\sqrt{f h} \partial_{r} \psi\right)=-2 \partial_{r} K,
$$

whose general solution of this equation is [5, 19]

$$
\psi=a t+\int \frac{b-2 K}{\sqrt{f h}} d r=a t+b r_{*}-\ln (f),
$$


where $a$ and $b$ are constants to be specified by imposing appropriate boundary condition, and $r_{*}$ is the tortoise coordinate defined by $r_{*}=\int d r / \sqrt{f h}=r+M^{2} /(4 r)+2 M \ln (r)+4 M \ln [1-$ $M /(2 r)]+$ const.

Using this solution for $\psi$ and its partial derivatives

$$
\partial_{t} \psi=a, \quad \partial_{r} \psi=(b-2 K) / \sqrt{f h},
$$

the energy-momentum tensor is calculated as

$$
\begin{aligned}
& T_{t t}=-\frac{1}{96 \pi}\left(a^{2}+b^{2}-4 K^{2}+8 \sqrt{f h} K^{\prime}\right), \\
& T_{t r}=T_{r t}=-\frac{a b}{48 \pi \sqrt{f h}}, \\
& T_{r r}=-\frac{1}{96 \pi f h}\left(a^{2}+b^{2}-4 K^{2}\right) .
\end{aligned}
$$

Introducing the Eddington-Finkelstein (EF) coordinate system $\{u, v\}$, where $u=t-r_{*}$ and $v=t+r_{*}$, all components of the anomaly induced stress tensor in the EF null frame are

$$
\begin{aligned}
T_{u u} & =\frac{1}{4}\left(T_{t t}-2 \sqrt{f h} T_{t r}+f h T_{r r}\right)=-\frac{1}{192 \pi}\left[(a-b)^{2}-4 K^{2}+4 \sqrt{f h} K^{\prime}\right] \\
& =-\frac{1}{192 \pi}\left[(a-b)^{2}-4 \kappa^{2}\right] \quad\left(r=r_{H}\right), \\
T_{u v} & =T_{v u}=\frac{1}{4}\left(T_{t t}-f h T_{r r}\right)=-\frac{1}{48 \pi} \sqrt{f h} K^{\prime}, \\
T_{v v} & =\frac{1}{4}\left(T_{t t}+2 \sqrt{f h} T_{t r}+f h T_{r r}\right)=-\frac{1}{192 \pi}\left[(a+b)^{2}-4 K^{2}+4 \sqrt{f h} K^{\prime}\right] \\
& =-\frac{1}{192 \pi}(a+b)^{2} \quad(r \rightarrow \infty) .
\end{aligned}
$$

It is known that the gravitational anomaly analysis selects the Unruh vacuum to be the relevant quantum state. So the appropriate boundary condition for our purpose should correspond to the Unruh vacuum, and such a boundary condition should be imposed on the auxiliary field $\psi$.

Therefore, to specify the unknown constants $a$ and $b$, we shall adopt the Unruh vacuum as the boundary condition that assumes there is no ingoing flux (incoming radiation) on past null infinity $\left(T_{v v}=0\right.$ as $\left.r \rightarrow \infty\right)$ and the energy-momentum tensor has to be regular on the future horizon $\left(T_{u u}=0\right.$ for $\left.r=r_{H}\right)$. That is

$$
T_{u u}=0 \quad\left(r=r_{H}\right), \quad T_{v v}=0 \quad(r \rightarrow \infty) .
$$

From this boundary condition, we obtain $a=-b= \pm \kappa$.

Thus, we get the explicit expression for the $(r, t)$-component of the energy-momentum tensor

$$
T_{t}^{r}=-\frac{a b}{48 \pi \sqrt{f / h}}=\frac{\kappa^{2}}{48 \pi \sqrt{f / h}},
$$

from which, the energy flux of Hawking radiation can be evaluated by taking the asymptotic limit $(r \rightarrow \infty)$. This yields

$$
T_{t}^{r}(r \rightarrow \infty)=\frac{\kappa^{2}}{48 \pi}
$$


On the other hand, $T_{t}^{r}$ diverges on the horizon $\left(T_{t}^{r} \rightarrow \infty\right.$ as $\left.r \rightarrow r_{H}\right)$, but $\sqrt{-g} T_{t}^{r}$ always remains finite everywhere,

$$
\sqrt{-g} T_{t}^{r}=\frac{\kappa^{2}}{48 \pi}
$$

This means that $\sqrt{-g} T_{t}^{r}$ is regular at the horizon.

It is quite interesting to examine the behavior of the auxiliary field $\psi$. The asymptotic requirement $b=-a$ yields $\psi \sim-b u$ as $r \rightarrow \infty, T_{\mu v}$ describes an outgoing radiation; whereas the regularity condition on the horizon $b=\kappa$ reveals that $\psi \sim-\kappa v$ as $r \rightarrow r_{H}, T_{\mu v}$ is finite on the future horizon and describes an ingoing flux of negative energy radiation. Both $\psi$ and $\nabla_{\mu} \psi$ are regular on the future horizon.

\subsection{Covariant energy-momentum tensor and Hawking flux}

We now turn to the covariant energy-momentum tensor given by [16, 21]

$$
\widetilde{T}_{v}^{\mu}=-\frac{1}{96 \pi}\left(\frac{1}{2} D^{\mu} \psi D_{v} \psi-D^{\mu} D_{v} \psi+\delta_{v}^{\mu} R\right)
$$

where the auxiliary field $\psi$ still obey the equation $\square \psi=R$, while $D_{\mu}$ is the chiral covariant differential operator defined by

$$
D_{\mu}=\nabla_{\mu}+\varepsilon_{\mu \nu} \nabla^{v}=\varepsilon_{\mu v} D^{v} .
$$

This covariant energy-momentum tensor simultaneously satisfies the conformal anomaly equation and the covariant anomaly equation as follows

$$
\widetilde{T}_{\mu}^{\mu}=-\frac{R}{48 \pi}, \quad \nabla_{\mu} \widetilde{T}_{v}^{\mu}=-\frac{1}{96 \pi} \varepsilon_{\mu v} \partial^{\mu} R .
$$

Inserting the explicit solution for $\psi$ and its chiral covariant derivatives

$$
D_{t} \psi=-\sqrt{f / h} D^{r} \psi=a-b+2 K,
$$

the $(r, t)$-component of the covariant energy-momentum tensor can be computed as

$$
\widetilde{T}_{t}^{r}=\frac{1}{192 \pi \sqrt{f / h}}\left[(a-b)^{2}-4 K^{2}+4 \sqrt{f h} K^{\prime}\right] .
$$

Taking the $r \rightarrow \infty$ limit with which $\sqrt{-g} \rightarrow 1$, we get the covariant form of the energy flux

$$
\widetilde{T}_{t}^{r}(r \rightarrow \infty)=\frac{1}{192 \pi}(a-b)^{2} .
$$

From the requirement that the energy flux at spatial infinity should be $\kappa^{2} /(48 \pi)$, we obtain $a=b \pm 2 \kappa$.

Substituting this condition back to the expression of the covariant stress tensor, we find

$$
\begin{aligned}
\widetilde{T}_{t}^{r} & =\frac{1}{48 \pi \sqrt{f / h}}\left(\kappa^{2}-K^{2}+\sqrt{f h} K^{\prime}\right) \\
& =\frac{\kappa^{2}}{48 \pi} \frac{(1-M / 2 r)^{3}}{(1+M / 2 r)^{9}}\left(1+\frac{6 M}{r}+\frac{35 M^{2}}{2 r^{2}}+\frac{3 M^{3}}{2 r^{3}}+\frac{M^{4}}{16 r^{4}}\right) .
\end{aligned}
$$

At the horizon, $\widetilde{T}_{t}^{r}$ approaches to zero as $r \rightarrow r_{H}$. This fact supports the validity of the regularity condition proposed in [4], which demands that $\widetilde{T}_{t}^{r}$ should vanish at the horizon. 
It suggests the covariant regularity condition $\left(\widetilde{T}_{t}^{r}=0\right.$ at $\left.r=r_{H}\right)$ can be imposed as usual. In other words, the validity of the anomaly analysis at the horizon can be guaranteed so that the gravitational anomaly cancellation method can be safely extrapolated to the case when $\sqrt{-g}=0$ at $r=r_{H}$.

On the other hand, if we consider the near-horizon behavior

$$
\sqrt{-g} \widetilde{T}_{t}^{r}=\frac{1}{192 \pi}\left[(a-b)^{2}-4 K^{2}+4 \sqrt{f h} K^{\prime}\right]=\frac{1}{192 \pi}\left[(a-b)^{2}-4 \kappa^{2}\right] \quad\left(r=r_{H}\right),
$$

we can see that $\sqrt{-g} \widetilde{T}_{t}^{r}$ is apparently well-behaved everywhere. In particular, the above condition $(a=b \pm 2 \kappa)$ makes $\sqrt{-g} \widetilde{T}_{t}^{r} \equiv 0$ become an identity at the horizon. Conversely, if we let $\sqrt{-g} \widetilde{T}_{t}^{r}=0$, then we arrive at $\widetilde{T}_{t}^{r}(r \rightarrow \infty)=\kappa^{2} /(48 \pi)$.

Finally, it should be pointed out that the general expression for the covariant stress tensor

$$
\sqrt{-g} \widetilde{T}_{t}^{r}=\frac{1}{48 \pi}\left(\kappa^{2}-K^{2}+\sqrt{f h} K^{\prime}\right)=\left\{\begin{array}{ll}
0 & \left(r=r_{H}\right) \\
\frac{\kappa^{2}}{48 \pi} & (r \rightarrow \infty)
\end{array},\right.
$$

is identical to the result derived by using the anomaly cancellation method in the next section, establishing the equivalence of these two methods.

As a comparison with the energy-momentum tensor derived in the last subsection, the components of the covariant energy-momentum tensor are given by

$$
\begin{aligned}
& \widetilde{T}_{t t}=-\frac{1}{48 \pi}\left(\kappa^{2}-K^{2}+2 \sqrt{f h} K^{\prime}\right), \\
& \widetilde{T}_{t r}=\frac{1}{48 \pi \sqrt{f h}}\left(\kappa^{2}-K^{2}+\sqrt{f h} K^{\prime}\right), \\
& \widetilde{T}_{r r}=-\frac{1}{48 \pi f h}\left(\kappa^{2}-K^{2}\right) .
\end{aligned}
$$

Transformed into the EF null frame, they are

$$
\begin{aligned}
& \widetilde{T}_{u u}=-\frac{1}{48 \pi}\left(\kappa^{2}-K^{2}+\sqrt{f h} K^{\prime}\right)=-\frac{\kappa^{2}}{48 \pi} \quad(r \rightarrow \infty), \\
& \widetilde{T}_{u v}=-\frac{1}{96 \pi} \sqrt{f h} K^{\prime}, \\
& \widetilde{T}_{v v}=0 .
\end{aligned}
$$

\subsection{Comparison with results in the standard coordinates}

We now compare the results obtained in the above two subsections with those derived in the two-dimensional standard Schwarzschild coordinates (29) with the effective metric given by [4]

$$
d s^{2}=-\left(1-\frac{2 M}{\hat{r}}\right) d t^{2}+\frac{d \hat{r}^{2}}{1-2 M / \hat{r}}
$$

with $f=h=1-2 M / \hat{r}$. At the horizon $\left(\hat{r}_{H}=2 M\right)$, the surface gravity is $\kappa=1 /(4 M)$.

The previous analysis can be naively applied to this case. Now we have $\sqrt{-\hat{g}}=1$ and

$$
K=\frac{1}{2} \partial_{\hat{r}} f=\frac{M}{\hat{r}^{2}}, \quad R=-2 \partial_{\hat{r}} K=\frac{4 M}{\hat{r}^{3}} .
$$


Note also that the tortoise coordinate is replaced by $\hat{r}_{*}=\int d \hat{r} / f=\hat{r}+2 M \ln (\hat{r}-2 M)+$ const.

Then the consistent and covariant energy-momentum tensors are, respectively, given by [5, 16]

$$
\begin{aligned}
T_{t}^{\hat{r}} & =\frac{\kappa^{2}}{48 \pi}, \\
\widetilde{T}_{t}^{\hat{r}} & =\frac{1}{48 \pi}\left(\kappa^{2}-K^{2}-\frac{1}{2} f R\right) \\
& =\frac{\kappa^{2}}{48 \pi}\left(1+\frac{4 M}{\hat{r}}+\frac{12 M^{2}}{\hat{r}^{2}}\right)\left(1-\frac{2 M}{\hat{r}}\right)^{2} \\
& = \begin{cases}0 & \left(\hat{r}=\hat{r}_{H}\right), \\
\frac{\kappa^{2}}{48 \pi} & (\hat{r} \rightarrow \infty) .\end{cases}
\end{aligned}
$$

This means that the validity of the covariant regularity boundary condition $\widetilde{T}_{t}^{\hat{r}}=0$ at the horizon is justified by the effective action method [5, 16, 18, 19] in the case of the twodimensional Schwarzschild black hole (29).

In the above analysis, we have obtained a correct Hawking temperature $T=1 /(8 \pi M)$ for the effective metric (24). On the contrary, if we directly apply the effective action method to the case of the in-equivalently reduced metric (26), we will get a temperature $T=1 /(16 \pi M)$, which is half of the correct one originally derived by Hawking. The same incorrect temperature can also be deduced by the RW's method. This unexpected result can be further excluded by taking into account the covariance of the energy-momentum tensor under the isotropic coordinate transformation.

As mentioned before, the two-dimensional effective metric (24) can be obtained from the one (29) in the standard coordinates via the isotropic coordinate transformation (2), while the two-dimensional line element (26) can not. The general covariance implies that the result derived from the latter metric (26) is non-physical.

Under this isotropic coordinate transformation $\hat{r}=r[1+M /(2 r)]^{2}$, the consistent and covariant energy-momentum tensors transform as

$$
\begin{aligned}
& T_{t}^{\hat{r}}=\frac{\partial \hat{r}}{\partial r} T_{t}^{r}=\left(1-\frac{M^{2}}{4 r^{2}}\right) T_{t}^{r}, \\
& \widetilde{T}_{t}^{\hat{r}}=\frac{\partial \hat{r}}{\partial r} \widetilde{T}_{t}^{r}=\left(1-\frac{M^{2}}{4 r^{2}}\right) \widetilde{T}_{t}^{r} .
\end{aligned}
$$

Using $\sqrt{-g}=1-M^{2} /\left(4 r^{2}\right)$ and noting $\sqrt{-\hat{g}}=1$, we find that

$$
\begin{aligned}
& T_{t}^{\hat{r}}=\sqrt{-g} T_{t}^{r}=\frac{\kappa^{2}}{48 \pi}, \\
& \widetilde{T}_{t}^{\hat{r}}=\sqrt{-g} \widetilde{T}_{t}^{r}=\frac{1}{48 \pi}\left(\kappa^{2}-K^{2}-\frac{1}{2} f R\right),
\end{aligned}
$$

which means both $\sqrt{-\hat{g}} T_{t}^{\hat{r}}=\sqrt{-g} T_{t}^{r}$ and $\sqrt{-\hat{g}} \widetilde{T}_{t}^{\hat{r}}=\sqrt{-g} \widetilde{T}_{t}^{r}$ are not only covariant but also conformal invariant in two dimensions.

The regularity boundary condition [4] at the horizon naturally leads to

$$
\sqrt{-g} \widetilde{T}_{t}^{r}=\widetilde{T}_{t}^{\hat{r}}=0
$$


which is in accordance with our previous result. This fact reflects the covariance and the universality of the effective action method.

To end up with this section, our main results can be summarized in one word, that is, $\sqrt{-g} T_{t}^{r}$ is regular everywhere and the regularity boundary condition $\widetilde{T}_{t}^{\hat{r}}=0$ at the horizon has been obviously verified. This conclusion suggests that the gravitational anomaly analysis can work well at the horizon.

\section{Anomalies and Hawking temperatures}

In this section, we will include the RW' method extended in [8] for self-consistence. The general case considered in [8] for the two-dimensional black hole is non-extremal, including the extremal black hole as a special subcase. It is of particular interest to consider the metric of the two-dimensional Schwarzschild black hole in the isotropic coordinates. In this case, the metric determinant $\sqrt{-g}=0$ at $r=r_{H}, \sqrt{-g}=1$ as $r \rightarrow \infty$, and $\sqrt{-g} \neq 1$ elsewhere. Our previous analysis carried through in [8] had obviously overlooked this case.

Because the metric determinant equals to zero at the horizon, the gravitational anomaly method will become problematic there. Therefore a careful investigation is needed for the reliability of the anomaly analysis at the horizon. However, we have argued in the preceding section that $\sqrt{-g} T_{t}^{r}$ remains finite and $\widetilde{T}_{t}^{\hat{r}}$ is indeed equal to zero at the horizon, which ensures the covariant regularity boundary condition $\widetilde{T}_{t}^{\hat{r}}=0$ at the horizon can be imposed as usual. Therefore, the anomaly analysis is reasonable at the horizon, and the RW's method is applicable to the case considered here. In the subsequent analysis, we will apply the anomaly cancellation method and the effective action method to derive the Hawking temperature in the background space-time (26) recast into the general form (16).

\subsection{Consistent anomaly and covariant boundary condition}

The gravitational anomaly method proposed in [3] interprets Hawking radiation as a compensating energy flux that cancels the consistent or covariant gravitational anomaly at the horizon. The main idea goes as follows. Near the horizon, when omitting the ingoing modes that can not affect the physics outside of the horizon at classical level, the effective two-dimensional field theory becomes chiral and exhibits an anomaly. Therefore the quantum effective action breaks down the symmetry under the diffeomorphism transformation, which contradicts the fact that the underlying theory is covariant. As a result, in order to preserve the covariance of theory at quantum level, Hawking radiation must be included to cancel the anomaly near the horizon. In this method, the contribution from the dilaton can be neglected thanks to the static space-time.

In the following, our starting point for the consistent anomaly analysis will be based upon the general effective metric (16). Let us consider the effective field theory in the twodimensional background space-time (16). We divide the region outside of the horizon into two parts: the near-horizon region $\left[r_{H}, r_{H}+\varepsilon\right]$ and the far-away region $\left[r_{H}+\varepsilon,+\infty\right)$, where $\varepsilon$ will be taken the $\varepsilon \rightarrow 0$ limit ultimately. In the region $\left[r_{H}, r_{H}+\varepsilon\right]$, the theory becomes chiral 
after neglecting the classically irrelevant ingoing modes and the energy-momentum tensor there satisfies the anomaly equation.

It is well known that the minimal form of the consistent gravitational anomaly for righthanded fields reads [22]

$$
\nabla_{\mu} T_{v}^{\mu}=\frac{1}{96 \pi \sqrt{-g}} \varepsilon^{\beta \alpha} \partial_{\alpha} \partial_{\mu} \Gamma_{v \beta}^{\mu}=\frac{1}{\sqrt{-g}} \partial_{\mu} N_{v}^{\mu}
$$

while the covariant anomaly takes the form

$$
\nabla_{\mu} \widetilde{T}_{\nu}^{\mu}=\frac{-1}{96 \pi} \sqrt{-g} \varepsilon_{\mu \nu} \partial^{\mu} R=\frac{1}{\sqrt{-g}} \partial_{\mu} \widetilde{N}_{\nu}^{\mu}
$$

where $\varepsilon^{\mu v}$ is the antisymmetric tensor density with the upper and the lower case $\varepsilon^{t r}=\varepsilon_{r t}=1$.

Solving the anomaly equations in terms of the effective background metric (16), we get the $(r, t)$-components of $N_{v}^{\mu}$ and $\widetilde{N}_{v}^{\mu}$

$$
\begin{aligned}
& N_{t}^{r}=\frac{1}{192 \pi}\left(h f^{\prime \prime}+f^{\prime} h^{\prime}\right), \\
& \widetilde{N}_{t}^{r}=\frac{1}{96 \pi}\left(h f^{\prime \prime}+\frac{1}{2} f^{\prime} h^{\prime}-\frac{h}{f} f^{\prime 2}\right)=\frac{1}{48 \pi}\left(\sqrt{f h} K^{\prime}-K^{2}\right) .
\end{aligned}
$$

Introducing two scalar (top hat and step) functions $\Theta(r)=\Theta\left(r-r_{H}-\varepsilon\right)$ and $H(r)=$ $1-\Theta(r)$, the total energy-momentum tensor can be expressed as

$$
T_{v}^{\mu}=T_{(O) v}^{\mu} \Theta(r)+T_{(H) v}^{\mu} H(r),
$$

where $T_{(O) v}^{\mu}$ is the covariantly conserved stress tensor satisfying $\nabla_{\mu} T_{(O) v}^{\mu}=0$, and $T_{(H) v}^{\mu}$ must obey Eq. (74). Integrating both equations, we get

$$
\begin{aligned}
& \sqrt{-g} T_{(O) t}^{r}=a_{O}, \\
& \sqrt{-g} T_{(H) t}^{r}=a_{H}+N_{t}^{r}-N_{t}^{r}\left(r_{H}\right),
\end{aligned}
$$

where $a_{O}$ and $a_{H}$ are two integration constants, and $a_{O}$ is the value of the energy-momentum tensor flux at spatial infinity, representing the observed Hawking radiation from the horizon. Note that both $T_{(O) t}^{r}$ and $T_{(H) t}^{r}$ diverges on the horizon because $\sqrt{-g}=0$ there, but $\sqrt{-g} T_{(O) t}^{r}$ and $\sqrt{-g} T_{(H) t}^{r}$ remain finite at the horizon.

Our main task is to fix $a_{O}$. In order to do so, let us consider the $v=t$ component of the conservation law

$$
\sqrt{-g} \nabla_{\mu} T_{t}^{\mu}=\partial_{r}\left[\sqrt{-g} T_{t}^{r}\right]=\partial_{r}\left[N_{t}^{r} H(r)\right]+\left[N_{t}^{r}+\sqrt{-g}\left(T_{(O) t}^{r}-T_{(H) t}^{r}\right)\right] \delta\left(r-r_{H}\right) .
$$

The first term can be cancelled by the quantum effect of the ingoing modes. One can make the energy-momentum tensor free of anomaly by re-defining a new one via $\bar{T}_{t}^{r}=$ $T_{t}^{r}-N_{t}^{r} H(r) / \sqrt{-g}$. Therefore, in order to keep the covariance of the underlying theory, we only need to make the coefficient of the second term disappear at the horizon

$$
\left.\sqrt{-g}\left(T_{(O) t}^{r}-T_{(H) t}^{r}\right)\right|_{r_{H}}+N_{t}^{r}\left(r_{H}\right)=0
$$

that is to say,

$$
a_{O}=a_{H}-N_{t}^{r}\left(r_{H}\right) .
$$

This equation can not fix $a_{O}$ completely unless the value of the constant $a_{H}$ is known. 
In Ref. [4], a covariant regularity condition is imposed to demand that the covariant energy-momentum tensor should vanish at the horizon $\left(\widetilde{T}_{t}^{r}=0\right.$ at $\left.r=r_{H}\right)$. Now that we have explicitly shown in the last section for the case considered here, $\widetilde{T}_{t}^{r}$ is indeed equal to zero at the horizon, the same boundary condition still can be imposed so as to be capable of applying the RW's method. As a result, we have $\sqrt{-g}=\widetilde{T}_{t}^{r}=0=\sqrt{-g} \widetilde{T}_{t}^{r}$ at the horizon.

Since the covariant and consistent anomalies are related by [8]

$$
\sqrt{-g} \widetilde{T}_{t}^{r}=\sqrt{-g} T_{t}^{r}+\widetilde{N}_{t}^{r}-N_{t}^{r}=\sqrt{-g} T_{t}^{r}+\frac{h}{192 \pi f}\left(f f^{\prime \prime}-2 f^{\prime 2}\right),
$$

as a consequence, the regularity condition yields

$$
a_{H}=N_{t}^{r}\left(r_{H}\right)-\widetilde{N}_{t}^{r}\left(r_{H}\right) .
$$

Therefore we get the expression of Hawking flux by taking the asymptotic limit

$$
T_{(O) t}^{r}(r \rightarrow \infty)=a_{O}=-\widetilde{N}_{t}^{r}\left(r_{H}\right)=\frac{\kappa^{2}}{48 \pi},
$$

which is obtained via the cancellation of the consistent gravitational anomaly at the horizon. It agrees with the results found in [12, 14].

The expression for the consistent energy-momentum tensor

$$
\sqrt{-g} T_{(O) t}^{r}=-\widetilde{N}_{t}^{r}\left(r_{H}\right)=\frac{\kappa^{2}}{48 \pi}
$$

is in exact accordance with the result obtained in the last section. This fact verifies the equivalence between the RW's method and the effective action method.

We now point out the difference between the above result and the previous one given in $[8]$

$$
a_{O}=N_{t}^{r}\left(r_{H}\right)=\left.\frac{1}{192 \pi}\left(h f^{\prime \prime}+f^{\prime} h^{\prime}\right)\right|_{r_{H}} .
$$

In Ref. [8], we have considered the general non-extremal black hole case in which $f\left(r_{H}\right)=$ $h\left(r_{H}\right)=0$. This means that the functions $f(r)$ and $h(r)$ are assumed to have the same asymptotic behaviors, namely, they simultaneously behave like $\left(r-r_{H}\right)$ or $\left(r-r_{H}\right)^{2}$ near the horizon. It is not difficult to find that the relation $a_{O}=N_{t}^{r}\left(r_{H}\right)=-\widetilde{N}_{t}^{r}\left(r_{H}\right)$ indeed and only holds true in such cases.

\subsection{Covariant anomaly only}

Recently, the authors in [12] suggested it is much cleaner to use only the covariant anomaly to derive the Hawking flux. In the above subsection, we have adopted the model proposed in [4] to calculate the energy-momentum flux through the cancellation of the consistent anomaly, but to fix it we have imposed a regular condition that requires $\widetilde{T}_{t}^{r}=0$ at the horizon. This implies that we have adopted two different anomalies to obtain the Hawking flux. By contrast, the only input advocated in [12] is the covariant anomaly. Nevertheless, we can see below that the result is unchanged. 
In the exterior region, there is no anomaly and the covariant energy-momentum tensor is conserved, $\nabla_{\mu} \widetilde{T}_{(O) v}^{\mu}=0$. Near the horizon, the energy-momentum tensor obeys the covariant anomaly equation, $\sqrt{-g} \nabla_{\mu} \widetilde{T}_{(H) v}^{\mu}=\partial_{v} \widetilde{N}_{v}^{\mu}$. Solving both equations for the $v=t$ component,

$$
\begin{aligned}
& \partial_{r}\left[\sqrt{-g} \widetilde{T}_{(O) t}^{r}\right]=0, \\
& \partial_{r}\left[\sqrt{-g} \widetilde{T}_{(H) t}^{r}\right]=\partial_{r} \widetilde{N}_{t}^{r},
\end{aligned}
$$

yields the solutions

$$
\begin{aligned}
& \sqrt{-g} \widetilde{T}_{(O) t}^{r}=\widetilde{a}_{O}, \\
& \sqrt{-g} \widetilde{T}_{(H) t}^{r}=\widetilde{a}_{H}+\widetilde{N}_{t}^{r}-\widetilde{N}_{t}^{r}\left(r_{H}\right),
\end{aligned}
$$

where $\widetilde{a}_{O}$ and $\widetilde{a}_{H}$ are two integration constants.

Writing the covariant stress tensor as a sum of two combinations $\widetilde{T}_{v}^{\mu}=\widetilde{T}_{(O) v}^{\mu} \Theta(r)+$ $\widetilde{T}_{(H) v}^{\mu} H(r)$, we find

$$
\sqrt{-g} \nabla_{\mu} \widetilde{T}_{t}^{\mu}=\partial_{r}\left[\sqrt{-g} \widetilde{T}_{t}^{r}\right]=\partial_{r}\left[\widetilde{N}_{t}^{r} H(r)\right]+\left[\widetilde{N}_{t}^{r}+\sqrt{-g}\left(\widetilde{T}_{(O) t}^{r}-\widetilde{T}_{(H) t}^{r}\right)\right] \delta\left(r-r_{H}\right) .
$$

The first term has to be cancelled by the quantum effect of the incoming modes. As before, it implies the existence of a Wess-Zumino term modifying the covariant energy-momentum tensor as $\bar{T}_{t}^{r}=\widetilde{T}_{t}^{r}-\widetilde{N}_{t}^{r} H(r) / \sqrt{-g}$ which is anomaly free provided the coefficient of the last term vanishes. This yields the condition

$$
\widetilde{a}_{O}=\widetilde{a}_{H}-\widetilde{N}_{t}^{r}\left(r_{H}\right) .
$$

As before, the constant $\widetilde{a}_{H}$ can be fixed by the covariant regularity boundary condition $\widetilde{T}_{t}^{r}=0$ at the horizon, which gives $\widetilde{a}_{H}=0$. Hence the covariant energy-momentum tensor flux is given by

$$
\widetilde{T}_{(O) t}^{r}(r \rightarrow \infty)=\widetilde{a}_{O}=-\widetilde{N}_{t}^{r}\left(r_{H}\right)=\frac{\kappa^{2}}{48 \pi} .
$$

This is just the energy flux from black body radiation.

Finally, the covariant energy-momentum tensors can be expressed as

$$
\begin{aligned}
& \sqrt{-g} \widetilde{T}_{(O) t}^{r}=-\widetilde{N}_{t}^{r}\left(r_{H}\right)=\frac{\kappa^{2}}{48 \pi}, \\
& \sqrt{-g} \widetilde{T}_{(H) t}^{r}=\widetilde{N}_{t}^{r}-\widetilde{N}_{t}^{r}\left(r_{H}\right)=\frac{1}{48 \pi}\left(\kappa^{2}-K^{2}+\sqrt{f h} K^{\prime}\right),
\end{aligned}
$$

which coincides with the results obtained in the last section. This indicates that both the gravitational anomaly method [3, 4, 12] and the effective action method [5, 16, 19] give the same consistent results. Thus the equivalence between these two approaches is generally justified.

On the other hand, the above anomaly analysis can be directly applied to the effective metric (29) in the standard coordinates and the obtained results coincide with those obtained in [3, 4, 16]. Moreover, we find that $\sqrt{-\hat{g}} T_{t}^{\hat{r}}=\sqrt{-g} T_{t}^{r}$ and $\sqrt{-\hat{g}} \widetilde{T}_{t}^{\hat{r}}=\sqrt{-g} \widetilde{T_{t}^{r}}$ are invariant under the isotropic coordinate transformation. Combined with these results, it can be concluded that both methods are generally covariant and universal, supporting the universality of the Hawking radiation. 


\subsection{Hawking temperatures of the isotropic Schwarzschild black hole}

With the previous preparation in hand, it is now a position to apply the gravitational anomaly method and the effective action method to reproduce the Hawking temperature of a Schwarzschild black hole in the isotropic coordinates. First of all, according to the spirit of the RW's method, in order to cancel the gravitational anomaly at the horizon so that the general covariance can be restored at the quantum level, one must identify the Hawking flux with the thermal flux of two-dimensional black body radiation at a temperature $T=\kappa /(2 \pi)$. Next, the effective action method directly adopts the anomaly induced stress tensors to calculate the energy flux at spatial infinity. As we have seen before, these two methods are equivalent and give the universal expression for the Hawking flux: $\left.-\widetilde{N}_{t}^{r}\left(r_{H}\right)=\kappa^{2} /(48 \pi)\right)$, which can be identical with the energy flux $\left(\Phi=\pi T^{2} / 12\right)$ of two-dimensional black body radiation at the Hawking temperature. This means that we can use the relation $\Phi=-\widetilde{N}_{t}^{r}\left(r_{H}\right)$ to determine the Hawking temperature of the isotropic Schwarzschild black hole.

Now we are ready to explicitly calculate the Hawking temperature of the Schwarzschild black hole in the isotropic coordinates. As mentioned before, the dimension reduction procedure yields two classes of two-dimensional effective metrics: the conformal equivalent and the in-equivalent ones. Two typical representatives for them are given by the twodimensional metrics (24) and (26), respectively. Both the RW's method and the effective action method are viable and can be applied to these two cases.

First, let us begin with the equivalent classes. The two-dimensional effective metric (24) is just the $(t-r)$-sector of the original four-dimensional isotropic Schwarzschild spacetime. In this case, the dimension reduction keeps the rank of the singularity unchanged. Since the metric determinant is $\sqrt{-g}=1-M^{2} /\left(4 r^{2}\right)$, it will vanish at the horizon, causing a doubt about the reliability of a direct application of the RW's method to this case. Using the effective action method, we have obviously verified that $\sqrt{-g} T_{t}^{r}$ is finite and $\widetilde{T}_{t}^{r}$ vanishes at the horizon, showing that the covariant regularity condition $\widetilde{T}_{t}^{r}=0$ at the horizon can be imposed as usual. This in turn indicates that the validity of the anomaly analysis at the horizon can be guaranteed. Applying the RW's method and the effective action method to this case, we can derive a temperature $T=1 /(8 \pi M)$, which is exactly equal to the correct one originally given by Hawking.

Next, we turn to consider the two-dimensional background metric (26) for the inequivalent classes. In this case, we have $f(r)=h(r)=[1-M /(2 r)] /[1+M /(2 r)]^{3}$ and $\sqrt{-g}=1$. Both the anomaly analysis and the effective action method are directly applicable to calculate the Hawking flux. At the horizon $\left(r_{H}=M / 2\right)$, due to $f\left(r_{H}\right)=h\left(r_{H}\right)=0$, the energy flux is given by $\Phi=f^{\prime 2}\left(r_{H}\right) /(192 \pi)$. This will give a temperature $T=1 /(16 \pi M)$, which is only one-half of the correct Hawking temperature. The reason for this is that the rank of the singularity has been changed in the process of dimension reduction.

Therefore, for the conformal equivalent effective line elemnet(24), both methods can reproduce the correct Hawking temperature, while they can not do so for the in-equivalent reduced line element (26). The different results for the derived temperatures arise from the different choice of a conformal factor in the process of dimensional reduction, since it 
can have an important effect on the order of the zeros of the component $g_{t t}$ in the resultant two-dimensional effective metric. The discrepancy of the obtained results serve a technical warning on how to obtain the physically equivalent reduced metric in the two-dimensional effective theory.

Finally, taking into account the general covariance of the energy-momentum tensor under coordinate transformations, we can further exclude the undesired result for the Hawking temperature with a $1 / 2$ factor in the case of the in-equivalent reduced metric (26). The reason for this is because that the energy-momentum tensor is covariant under the isotropic coordinate transformation (2); both the effective action method and the RW's method are covariant. Furthermore, the two-dimensional effective metric (24) can be obtained from the one (29) in the standard coordinates via the isotropic coordinate transformation (2), while the two-dimensional line element (26) can not. So the general covariance implies that only for the conformal equivalent classes can one derive the expected temperature as that given by Hawking.

\section{Concluding remarks}

In this paper, we have utilized the effective action method and the gravitational anomaly method to derive the Hawking temperature of a Schwarzschild black hole in the isotropic coordinates. Our motivation is originated from the following facts: First of all, Hawking radiation is a universal quantum phenomenon which relies merely on the property of the horizon and is unrelated with a concrete coordinate system. Correspondingly, the Hawking temperature should have a unique value, independent of the concrete choice of different coordinates. In other words, the Hawking temperature should have the same value although the metric can be expressed in different forms by adopting different coordinate systems. Next, the RW's method is universal since the derivation of Hawking radiation via the viewpoint of the anomaly cancellation is only dependent on the anomaly taking place at the horizon. To preserve general covariance, it requires an outgoing thermal flux to eliminate the gravitational anomaly at the horizon. One can anticipate the effective action method to be universal also. Third, both methods are manifestly generally covariant. Therefore, it is expected that these two methods can deal with Hawking radiation in different coordinate settings and give the same consistent result. That is, if both methods are successful to derive the Hawking temperature of a Schwarzschild black hole in the standard coordinate system, the result obtained by these methods should be unchanged in a different coordinate system (here the isotropic coordinates).

Since both methods work within the two-dimensional effective field theory, so a key trick is how to reduce the higher-dimensional space-times to two dimensions. The dimension reduction procedure plays a crucial role in deriving the resultant two-dimensional effective background metrics so that they belong to the physically equivalent classes.

The Schwarzschild black hole in the isotropic coordinates displays a lot of distinct characters that differ from the standard one. It also provides an obvious example that one has to deal with the case in which not only $\sqrt{-g} \neq 1$ but also $\sqrt{-g}=0$ at the horizon for the 
conformal equivalent reduced metrics. Thanks to these intriguing features, it is of particular interest to check the effectiveness of the RW's method and the effective action method in the isotropic Schwarzschild space-time. Our results have demonstrated that both methods can derive the correct Hawking temperature for the conformal equivalent classes of the reduced effective metric (24).

The main results obtained in our paper are summarized as follows. (i) The dimensional reduction procedure yields two classes of typically different metrics. The physically equivalent reduced metrics are defined as those that neither change the rank of the zeros of the components $g_{t t}$ and $g^{r r}$ of the original space-time metric, nor flip their signs. These metrics are also conformal equivalent, up to a regular conformal factor that satisfies the conditions (22). (ii) The equivalence between the effective action method and the gravitational anomaly method are generally established. (iii) The covariance and the universality of both methods are obviously justified via the isotropic coordinate transformation. (iv) The covariant regularity boundary condition imposed in the RW's method has been verified by using the effective action method to explicitly calculate the covariant energy-momentum tensor, so the validity of RW's method has also been justified. (v) For the conformal equivalent reduced metric (24), both methods can successfully reproduce the standard value of the Hawking temperature for the isotropic Schwarzschild black hole. These results support not only the universality of these two methods but also that of Hawking radiation.

Based upon the RW's method and the effective action method, our discussions about the gravitational anomaly analysis presented in the isotropic coordinates are very general. They have an immediate application to the stringy black holes since a large classes of black hole solutions found in string theory are expressed in the isotropic coordinate system, especially for those supersymmetric black holes. It is interesting to check the effectiveness of these two methods in other different coordinate system case as well.

\section{Acknowledgements}

This work is partially supported by the Natural Science Foundation of China under Grant No. 10675051.

\section{References}

[1] Hawking S 1974 Nature (London) 248 30-31 Hawking S 1975 Commun. Math. Phys. 43 199-220

[2] Parikh M K and Wilczek F 2000 Phys. Rev. Lett. 85 5042-5045 (Preprint hep-th/9907001)

[3] Robinson S P and Wilczek F 2005 Phys. Rev. Lett. 95011303 (Preprint gr-qc/0502074)

[4] Iso S, Umetsu H and Wilczek F 2006 Phys. Rev. Lett. 96151302 (Preprint hep-th/0602146)

[5] Iso S, Umetsu H and Wilczek F 2006 Phys. Rev. D 74044017 (Preprint hep-th/0606018) Murata K and Soda J 2006 Phys. Rev. D 74044018 (Preprint hep-th/0606069) Iso S, Morita T and Umetsu H 2007 J. High Energy Phys. JHEP04(2007)068 (Preprint hep-th/0612286)

[6] Vagenas E C and Das S 2006 J. High Energy Phys. JHEP10(2006)025 (Preprint hep-th/0606077) Setare M R 2007 Euro. Phys. J. C 49 865-868 (Preprint hep-th/0608080)

Xiao K, Liu W B and Zhang H B 2007 Phys. Lett. B 647 482-485 (Preprint hep-th/0702199) 
Anomalies, effective action and Hawking radiation

Xu Z B and Chen B 2007 Phys. Rev. D 75024041 (Preprint hep-th/0612261)

Shin H and Kim W 2007 J. High Energy Phys. JHEP06(2007)012 (Preprint arXiv:0705.0265 [hep-th]) Kim W and Shin H 2007 J. High Energy Phys. JHEP07(2007)070 (Preprint arXiv:0706.3563 [hep-th])

Das S, Robinson S P and Vagenas E C 2008 Int. J. Mod. Phys. D 17 533-539 (Preprint arXiv:0705.2233 [hep-th])

[7] Jiang Q Q and Wu S Q 2007 Phys. Lett. B 647 200-206 (Preprint hep-th/0701002)

Jiang Q Q, Wu S Q and Cai X 2007 Phys. Lett. B 651 (2007) 58-64 (Preprint hep-th/0701048)

Jiang Q Q, Wu S Q and Cai X 2007 Phys. Lett. B 651 (2007) 65-70 (Preprint arXiv:0705.3871 [hep-th])

Jiang Q Q, Wu S Q and Cai X 2007 Phys. Rev. D 75064029 (Preprint hep-th/0701235)

Wu S Q 2007 Phys. Rev. D 76029904 (erratum)

Jiang Q Q 2007 Class. Quantum Grav. 24 4391-4406 (Preprint arXiv:0705.2068 [hep-th])

[8] Wu S Q and Peng J J 2007 Class. Quantum Grav. 24 5123-5138 (Preprint arXiv:0706.0983 [hep-th])

Peng J J and Wu S Q 2008 Chin. Phys. B 17 825-828 (Preprint arXiv:0705.1225 [hep-th])

[9] Miyamoto U and Murata K 2008 Phys. Rev. D 77024020 (Preprint arXiv:0705.3150 [hep-th])

Chen B and He W 2008 Class. Quantum Grav. 24 (at press) Preprint arXiv:0705.2984 [gr-qc]

[10] Murata K and Miyamoto U 2007 Phys. Rev. D 76084038 (Preprint arXiv:0707.0168 [hep-th])

[11] Ma Z Z 2007 Preprint arXiv:0709.3684[hep-th]

Huang C G, Sun J R, Wu X N and Zhang H Q 2007 Preprint arXiv:0710.4766 [hep-th]

Wu X N, Huang C G and Sun J R 2008 Phys. Rev. D 77 (at press) Preprint arXiv:0801.1347 [gr-qc]

He T M, Fan J H and Wang Y J 2008 Chin. Phys. B 172321

Zeng X X, Chen D Y and Yang S Z 2008 Chin. Phys. B 171629

Lin K, Chen S W and Yang S Z 2008 Int. J. Theor. Phys. 47 (at press)

[12] Banerjee R and Kulkarni S 2008 Phys. Rev. D 77024018 (Preprint arXiv:0707.2449 [hep-th])

[13] Wu S Q and Zhao Z Y 2007 Preprint arXiv:0709.4074 [hep-th]

[14] Peng J J and Wu S Q 2008 Phys. Lett. B 661 300-306 (Preprint arXiv:0801.0185 [hep-th])

Peng J J and Wu S Q 2008 Gen. Rel. Grav. 40 (at press) Preprint arXiv:0709.0167 [hep-th]

[15] Lin K, Zeng X X and Yang S Z 2008 Chin. Phys. Lett. 25 390-392

Zeng X X, Chen D Y and Yang S Z 2008 Gen. Rel. Grav. 40 (at press)

Han Y W, Zeng X X and Yang S Z 2008 Int. J. Theor. Phys. 47 (at press)

Zeng X X, Lin K and Yang S Z 2008 Int. J. Theor. Phys. 47 (at press)

[16] Banerjee R and Kulkarni S 2008 Phys. Lett. B 659 827-831 (Preprint arXiv:0709.3916 [hep-th])

Gangopadhyay S 2008 Phys. Rev. D 77064027 (Preprint arXiv:0712.3095 [hep-th])

Kulkarni S 2008 Preprint arXiv:0802.2456 [hep-th]

Gangopadhyay S and Kulkarni S 2008 Phys. Rev. D 77024038 (Preprint arXiv:0710.0974[hep-th])

[17] Breckenridge J C, Myers R C, Peet A W and Vafa C 1997 Phys. Lett. B 391 93-98 (Preprint hep-th/0902065)

[18] Mukhanov V, Wipf A and Zelnikov A 1994 Phys. Lett. B 332 283-291 (Preprint hep-th/9403018)

Frolov V P, Israel W and Solodukhin S N 1996 Phys. Rev. D 54 2732-2745 (Preprint hep-th/9602105)

Buric M, Radovanovic V and Mikovic A 1999 Phys. Rev. D 59084002 (Preprint gr-qc/9804083)

Lombardo F C, Mazzitelli F D and Russo J G 1999 Phys. Rev. D 59064007 (Preprint gr-qc/9808048)

Nojiri S and Odintsov S D 1998 Phys. Rev. D 57 2363-2371 (Preprint hep-th/9706143)

Kummer W and Vassilevich D V 1999 Ann. Phys. (Leipzig) 8 801-827 (Preprint /gr-qc/9907041)

[19] Balbinot R and Fabbri A 2003 Class. Quantum Grav. 20 5439-5454

Balbinot R and Fabbri A 1999 Phys. Rev. D 59044031 (Preprint hep-th/9807123)

Balbinot R and Fabbri A 1999 Phys. Lett. B 459 112-118 (Preprint gr-qc/9904034)

Balbinot R, Fabbri A and Shapiro I 1999 Nucl. Phys. B 559 301-319 (Preprint hep-th/9904162)

[20] Polyakov A M 1981 Phys. Lett. B 103 207-210

[21] Leutwyler H 1985 Phys. Lett. B $15365-69$

Leutwyler H 1985 Phys. Lett. B 155469 (erratum)

[22] Alvarez-Gaume L and Witten E 1984 Nucl. Phys. B 234 269-330

Bardeen W A and Zumino B 1984 Nucl. Phys. B 244 421-453 
Bertlmann R and Kohlprath E 2001 Ann. Phys. (N.Y.) 288 137-163 (Preprint hep-th/0011067) 